\title{
IKK-mediated CYLD phosphorylation and cellular redox activity
}

\author{
Adnan Erol ${ }^{*}($ )
}

\begin{abstract}
Oxidative stress is important in the development of obesity-related nephropathy (ORN). A causal relationship between IKK and ORN via CYLD-mediated inhibition of NRF2 has been described. However, contradictory explanations about the functioning of the mechanisms that will be effective in the pathogenesis require clarification.
\end{abstract}

Keywords: CYLD, IKK, NRF2, NF-KB, RIPK1

In a recent study published in the Journal, Chen et al. claimed that IKKs, NF-kB activating kinases, may reduce the cellular antioxidant capacity in obesity-related nephropathy (ORN) through inactivating one of the major deubiquitinases, CYLD (Chen et al. 2021). According to the authors of the study, ox-LDL-stimulated oxidative stress may activate IKK that, in turn, phosphorylates and inactivates the deubiquitinating activity of CYLD. Consequently, the increased ubiquitination and degradation of NRF2 may promote oxidative stress injury in ORN cells. Importantly, they found that NRF2 is molecularly encapsulated by "a series of high-molecular-weight species" and directly binds CYLD. Furthermore, the treatment of ORN cells with MG132, a proteasome inhibitor, resulted in the activation of NRF2-related transcription. Altogether, they concluded that IKK-mediated inactivation of CYLD may potentiate the oxidative stress injury through the increased NRF2 degradation.

Proteasomal degradation of NRF2 will reduce the antioxidant ability of the cell. However, concluding that CYLD will protect NRF2 degradation by reducing its polyubiquitination would be controversial. On the contrary, a previous study reported that CYLD may increase oxidative stress by inhibiting the anti-oxidative activity of NRF2, as they cited in their study (Wang et al. 2015).

*Correspondence: eroladnan@protonmail.com

Silivri-Istanbul, Turkey
CYLD, having deubiquitinase (DUB) activity, is a tumor suppressor that plays a key role in proliferation and cell death. CYLD negatively regulates the NF- $\mathrm{KB}$ signaling pathway by removing selectively Lysine (K)-63-linked and linear polyubiquitin chains but exhibits very little activity towards degradative K48-linked ubiquitin chains (Komander et al. 2009; Sato et al. 2015).

Previous studies with the tumor suppressor CYLD have described it as having an anti-inflammatory function, primarily because of its inhibitory effect on the NF- $\mathrm{KB}$ pathway. The Inhibition of CYLD increases resistance to apoptosis by activating NF- $\mathrm{KB}$, suggesting a mechanism through which loss of CYLD contributes to oncogenesis (Brummelkamp et al. 2003). Furthermore, CYLD is believed to promote apoptosis and programmed necrosis (necroptosis) by facilitating RIPK1 deubiquitination (Moquin et al. 2013). RIPK1 is a complex protein that possesses both a scaffolding pro-survival as well as a catalytic pro-death function. When RIPK1 is modified by K63- and M1-linked ubiquitins, it acts as a scaffold, independent of its kinase activity, to recruit some adaptor proteins, leading to the NF-kB-dependent transcription of pro-survival proteins (Li et al. 2020). Furthermore, the IKK complex that phosphorylates CYLD is also necessary for the induction of NF- $\mathrm{kB}$-dependent transcription. Until very recently, canonical IKK members, IKK $\alpha$ and IKK $\beta$, and non-canonical IKKe were believed to phosphorylate and inactivate the DUB activity of CYLD (Reiley et al. 2005; Hutti et al. 2009). However, a original author(s) and the source, provide a link to the Creative Commons licence, and indicate if changes were made. The images or other third party material in this article are included in the article's Creative Commons licence, unless indicated otherwise in a credit line to the material. If material is not included in the article's Creative Commons licence and your intended use is not permitted by statutory regulation or exceeds the permitted use, you will need to obtain permission directly from the copyright holder. To view a copy of this licence, visit http://creativecommons.org/licenses/by/4.0/. 
very recent study made the issue more complicated. The research revealed that IKK phosphorylation, paradoxically, stimulates rather than inhibits CYLD, promoting its DUB activity (Elliot et al. 2021). Thus, IKK-mediated CYLD phosphorylation deconjugates ubiquitin chains on RIPK1, increasing its kinase activity for the death signaling pathway. Phosphorylated CYLD also reduces resistance to apoptosis by the loss of scaffolding function of RIPK1 necessary to activate NF-KB-mediated transcription.

NRF2, the main regulator of the cytoprotective gene program, is a transcription factor that encodes not only antioxidant genes but also numerous detoxification enzymes that conjugate oxidation products. Canonically, KEAP1, an adaptor component of ubiquitin E3 ligase, constitutively ubiquitinates NRF2 with K48linked polyubiquitin chains. Consequently, KEAP1 promotes the proteasomal degradation of NRF2, thus keeping cellular NRF2 at a low level (Taguchi et al. 2012). On the other hand, in a noncanonical pathway, stimulated p62 can also bind to KEAP1. p62 is a stressinducible protein, which serves as an adaptor protein between selective autophagy and ubiquitin signaling. In the non-canonical KEAP1-NRF2 pathway, mTORC1mediated phosphorylation of $\mathrm{p} 62$ at serine-349 leads to the competitive binding with KEAP1. Following KEAP1 association with p62 and KEAP1-NRF2 dissociation, stabilized NRF2 translocates to the nucleus to induce its target genes. NRF2 positively regulates p62 gene expression; therefore, p62 is able to set up a positive feedback loop to activate NRF2, which in turn stimulates increased transcription of the p62 gene (Taguchi et al. 2012; Ichimura et al. 2013).

In the light of these data, it would be more rational to develop a new model between IKK-mediated CYLD phosphorylation and NRF2 activity. Under basal (unstimulated) conditions, KEAP1 constitutively ubiquitinates NRF2, leading to the rapid proteasomal degradation of NRF2 in the canonical KEAP1-NRF2 pathway (Fig. 1A). Following stimulation, CYLD undergoes phosphorylation at serine- 418 and serine- 568 residues catalyzed by either canonical IKKs (IKK $\alpha$ and IKK $\beta)$ or by the noncanonical IKKe, which may increase DUB activity of CYLD. The stimulated CYLD blocks the activation of mTORC1, increasing autophagy (Colombo et al. 2021). Consequently, the loss of phosphorylation of p 62 by mTORC 1

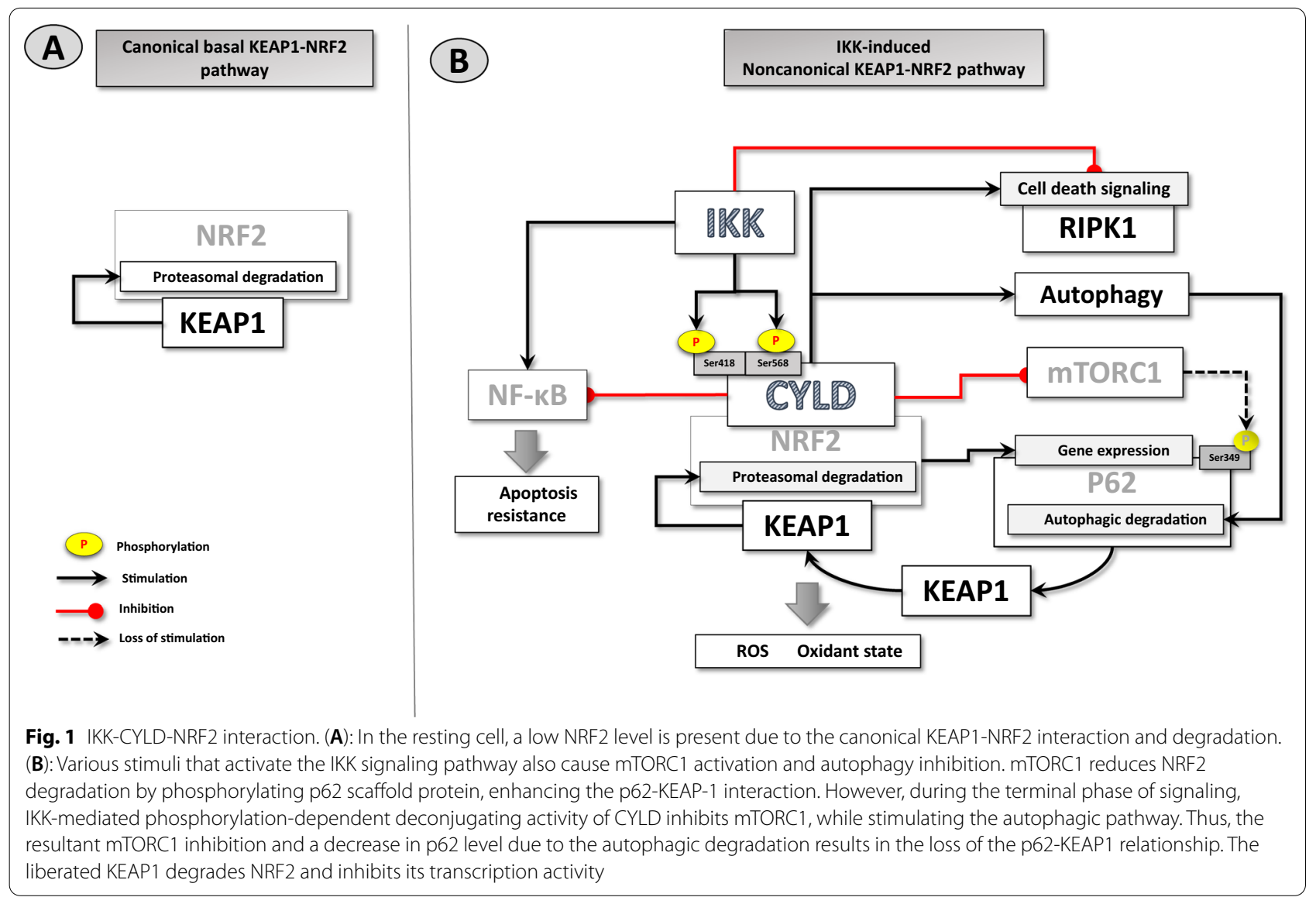


inhibits the interaction of p62 with KEAP1. Released KEAP1 can associate with NRF2, promoting NRF2 degradation, which also reduces the p62 level. In addition, CYLD stimulation will contribute to $\mathrm{p} 62$ reduction through its autophagic degradation. Altogether, reduced p62 levels can lead to the inhibition of NRF2- and NF$\mathrm{kB}$-mediated transcription, creating a positive cycle (Katsuragi et al. 2016) (Fig. 1B).

To summarize the results of IKK-stimulated CYLD activity: (1) decreases the affinity of p62 for KEAP1; thus, increases proteasomal degradation of NRF2 through the potentiated NRF2-KEAP1 association. This, in turn, will reduce the cellular redox potential, promoting oxidative stress and a reduction in p62 level as well; (2) increases autophagy, leading to the further p62 reduction; (3) stimulates RIPK1-mediated cell death (apoptosis and/or necroptosis); (4) inhibits apoptosis resistance, potentiating the RIPK1-mediated cell death.

ROS production was thought to be of key importance in ORN. NRF2 was generally thought to be a crucial cellular defense against oxidative stress (Chen et al. 2021). The findings of Chen et al., regarding NRF2 inhibition by IKK in ORN, which leads to increased oxidative stress, are important. However, their interpretation of the CLYD-NRF2 interaction does not seem to be compatible with the available data. This letter aims to reconcile conflicting results regarding the mechanism of NRF2 inhibition. Thus, it may make more sense for Chen et al. to use the model defined here, rather than their confusing implications, to explain the influence of CYLD on NRF2. This model can also be considered as a working mechanism for other chronic degenerative diseases in which IKK activation and oxidative stress are common denominators.

\section{Abbreviations}

CYLD: Cylindromatosis protein; IKK: IkB kinase; KEAP1: Kelch-like ECH-associated protein 1; mTORC 1: Mechanistic target of rapamycin; NF-KB: Nuclear Factor Kappa B; NRF2: NF-E2-related factor 2; ORN: Obesity-related nephropathy; ox-LDL: Oxidized low-density lipoprotein; RIPK1: Receptor-interacting protein kinase 1; ROS: Reactive oxygen species.

Acknowledgements

Not applicable.

Authors' contributions

The author read and approved the final manuscript.

Funding

No funding was received.

\section{Declarations}

Ethics approval and consent to participate Not applicable.
Consent for publication

Not applicable.

\section{Competing interests}

There are no commercial or other associations that might pose a conflict of interest.

Received: 23 December 2021 Accepted: 17 January 2022

Published online: 02 February 2022

\section{References}

Brummelkamp TR, et al. Loss of the cylindromatosis tumour suppressor inhibits apoptosis by activating NF-kappaB. Nature. 2003:424(6950):797-801. https://doi.org/10.1038/nature01811.

Chen YY, et al. IKB kinase promotes Nrf2 ubiquitination and degradation by phosphorylating cylindromatosis, aggravating oxidative stress injury in obesity-related nephropathy. Mol Med. 2021;27(1):137. https://doi.org/10. 1186/s10020-021-00398-w.

Colombo E, et al. The K63 deubiquitinase CYLD modulates autism-like behaviors and hippocampal plasticity by regulating autophagy and mTOR signaling. Proc Natl Acad Sci U S A. 2021;118(47): e2110755118. https:// doi.org/10.1073/pnas.2110755118.

Elliott PR, et al. Regulation of CYLD activity and specificity by phosphorylation and ubiquitin-binding CAP-Gly domains. Cell Rep. 2021;37(1): 109777. https://doi.org/10.1016/j.celrep.2021.109777.

Hutti JE, et al. Phosphorylation of the tumor suppressor CYLD by the breast cancer oncogene IKKepsilon promotes cell transformation. Mol Cell. 2009;34:461-72. https://doi.org/10.1016/j.molcel.2009.04.031.

Ichimura Y, et al. Phosphorylation of p62 activates the Keap1-Nrf2 pathway during selective autophagy. Mol Cell. 2013;51(5):618-31. https://doi.org/ 10.1016/.j.molcel.2013.08.003.

Komander D, et al. Molecular discrimination of structurally equivalent Lys 63-linked and linear polyubiquitin chains. EMBO Rep. 2009;10(5):466-73. https://doi.org/10.1038/embor.2009.55.

$\mathrm{Li}$ X, et al. Ubiquitination of RIPK1 regulates its activation mediated by TNFR1 and TLRs signaling in distinct manners. Nat Commun. 2020;11:6364. https://doi.org/10.1038/s41467-020-19935-y.

Moquin DM, McQuade T, Chan FK. CYLD deubiquitinates RIP1 in the TNFainduced necrosome to facilitate kinase activation and programmed necrosis. PLoS ONE. 2013;8(10): e76841. https://doi.org/10.1371/journal. pone.0076841.

Reiley W, et al. Regulation of the deubiquitinating enzyme CYLD by IkappaB kinase gamma-dependent phosphorylation. Mol Cell Biol. 2005;25(10):3886-95. https://doi.org/10.1128/MCB.25.10.3886-3895.2005.

Sato Y, et al. Structures of CYLD USP with Met1- or Lys63-linked diubiquitin reveal mechanisms for dual specificity. Nat Struct Mol Biol. 2015;22(3):222-9. https://doi.org/10.1038/nsmb.2970.

Taguchi K, et al. Keap1 degradation by autophagy for the maintenance of redox homeostasis. Proc Natl Acad Sci U S A. 2012;109(34):13561-6. https://doi.org/10.1073/pnas.1121572109.

Wang $\mathrm{H}$, et al. Deubiquitinating enzyme CYLD mediates pressure overloadinduced cardiac maladaptive remodeling and dysfunction via downregulating Nrf2. J Mol Cell Cardiol. 2015;84:143-53. https://doi.org/10.1016/j. yjmec.2015.04.012.

\section{Publisher's Note}

Springer Nature remains neutral with regard to jurisdictional claims in published maps and institutional affiliations. 